\title{
The influence of life history attributes and fishing pressure on the efficacy of marine reserves
}

\author{
Leah R. Gerber ${ }^{\mathrm{a}, *}$, Peter M. Kareivab ${ }^{\mathrm{b}}$, Jordi Bascompte ${ }^{\mathrm{a}, \mathrm{c}}$ \\ anational Center for Ecological Analysis and Synthesis, University of California, Santa Barbara, 735 State Street, Suite 300, \\ Santa Barbara, CA 93101-3351, USA \\ bNational Marine Fisheries Service, Northwest Fisheries Science Center, 2725 Montlake Boulevard East, Seattle, WA 98112, USA \\ 'Estación Biológica de Donana, CSIC Apdo. 1056, E-41013, E-41013 Sevilla, Spain
}

\begin{abstract}
Two key questions regarding "no-take" marine reserves are: (1) how effective are reserves likely to be, and (2) how does effectiveness vary with life history attributes and the relative size of reserves. To investigate these questions, we use a simple Ricker model that includes fishing, larval dispersal, and larval loss while in a planktonic pool, and that tracks protected and unprotected populations. We applied two different measures of reserve effectiveness to our simulation results. One metric was intended to reflect goals oriented towards conservation and the second was intended to reflect fishery enhancement goals. Both metrics compare the situation before reserves are established to after the reserve has been in place and a new equilibrium was reached. Yield effectiveness is defined as the total equilibrium annual harvest after reserves are established divided by the total annual harvest before reserves are established. Conservation effectiveness is defined as the average adult density inside the reserve divided by the average density in the same area prior to reserve establishment. A substantial fraction of the 5120 simulated parameter combinations representing different harvest rates and life history attributes went extinct in the absence of a reserve, and these scenarios leading to extinction could be predicted accurately (85\% aptly classified) simply on the basis of exploitation rate and population growth rate. Of the cases that did not go extinct, we compared the performance of reserves as measured by each effectiveness metric. Few of the cases (less than $8 \%$ ) produced effective reserves as measured in terms of increased harvest; whereas over half of the cases resulted in effective reserves as measured by conservation effectiveness. Moreover, the two measures of reserve effectiveness were only weakly correlated. Simple linear regression or polynomial regression could explain at most $23 \%$ of the variation in reserve effectiveness as measured by either metric. As expected, the size of the reserve area had a marked and typically negative effect on total annual yield, which suggests that while marine protected areas may do a good job of conserving protected populations, there will generally be pressure from the fishing community to keep them small because of their tendency to reduce total catch. \# 2002 Elsevier Science Ltd. All rights reserved.
\end{abstract}

Keywords: Marine reserves; Ricker model; Fishing pressure; Life history; Reserve size

\section{Introduction}

Biologists and conservationists are increasingly calling for the establishment of "no-take" zones in the marine environment so that populations of over-exploited marine species might recover (e.g. Myers and Mertz, 1997; Lauck et al., 1998). One interesting facet of pleas for marine reserves is that these reserves are typically quite small in area, and represent only a small portion

* Corresponding author. Tel.: +1-805-892-2517; fax: +1-805-8922510.

E-mail address: gerber@nceas.ucsb.edu (L.R. Gerber). of the total range of species (Allison et al., 1998). Thus, unlike standard fisheries regulations, which apply to entire stocks, the marine reserve approach is more similar in spirit to terrestrial nature reserves. In addition, although there has been an increase in the number of marine reserves designated to conserve marine species, there has not been a concomitant increase in our understanding of marine reserve theory. In many cases, resource managers and stakeholders expect to see major benefits in the short term after the establishment of a marine protected area (e.g. a 5-year timeframe was proposed to determine efficacy of no-take zones in the Channel Islands Marine Sanctuary). Thus, a key question 
regarding "no-take" marine reserves concerns the extent to which particular biological and harvest regimes combine to determine when the benefits of a reserve will be noticeable.

Existing single cohort models of marine reserves generally conclude that there is at least some potential for marine species to benefit from no-take zones (e.g. Polachek, 1990; DeMartini, 1993). The degree of effectiveness is dependent upon the level of fishing pressure (Gerber et al., 2001). In general, the benefits of reserves for stock recovery are greatest for overexploited stocks and are sensitive to how density-dependence impacts the population.

The question of impact of unfished areas on the yield to a fishery was first introduced by Beverton and Holt in their classic volume on fisheries management (Beverton and Holt, 1957, see also Guenette et al., 1998). Beverton and Holt (1957) approached the question of marine reserves by assuming that the original fishing effort would remain constant and be concentrated outside reserves (i.e. fishing mortality rate is multiplied by the ratio of fished areas to unfished areas). With this assumption, yield per recruit increased with increasing reserve area when reserve area was low, but decreased when reserved area was high, generating an optimal reserve fraction. Based on Beverton and Holt's model, Polachek (1990) used a two-component spatial model with movement between areas to consider the fate of a single cohort when only a portion of the population is vulnerable to fishing. Polachek (1990) found that reserves had a low potential for increasing yield per recruit beyond what was possible by controlling effort, but that higher eggs per recruit was possible, especially at lower movement rates. As movement rates increased, larger areas were needed for reserves to achieve gains. DeMartini (1993) used Polachek's (1990) model to assess the impact of reserves on three reef fish types with different life histories. DeMartini (1993) found that: (1) for the species with high movement rates, potential gains were negated by movement out of the reserve and subsequent capture; (2) for the species with low movement rates, gains were low because individuals rarely moved out of the reserves to be captured; and (3) that therefore gains were greatest for the species with moderate movement rates.

Other models explicitly include reproduction in their assessment of the effect of marine reserves on single populations. These models all lead to the general result that if fishing effort is high enough to cause recruitment overfishing in the absence of reserves, reserves can help prevent overfishing and promote a sustainable level of catch (e.g. Sladek Nowlis and Roberts, 1999). Quinn et al. (1993) used a stage-structured model with densitydependent fecundity and limited fertilization success of the broadcast spawner at low densities and a refuge for juveniles beneath the spine canopy of adults to represent the dynamics of red sea urchins distributed along the northern California coast. As fishing effort increased, in cases with no reserves or with reserves spaced too far apart, populations declined to zero abundance. This work suggests that reserves are most beneficial where local extinction due to fishing was high, and when configured as multiple reserves, spaced more closely than the average larval dispersal distance.

In this paper we explore a simple model that focuses on the degree to which life history attributes and level of fishing pressure combine to determine the value of marine reserves of variable size. One innovation of our analysis is to quantify reserve effectiveness using two different measures: how well populations build up inside the reserve as a result of removing harvest (conservation effectiveness, or CE), and the extent to which this buildup of fish populations inside a reserve spills over to sustain an increased harvest (yield effectiveness, or YE). In addition we identify the life history attributes in combination with levels of fishing pressure that determine whether populations go extinct. Our analyses focus on a single species, and a simple "single reserve" system. This simplification allows us to include a large mix of life history variants (different rates of population growth, migration rates, larval losses, and values for carrying capacity). Although structurally similar models have been applied to other marine reserve questions, none have sought to identify the combinations of life history attributes and no-take zones that at least minimally produce some measurable conservation or harvest benefit. In contrast, most existing marine reserve theory aims at identifying so-called "optimal reserve design". While this may be intellectually satisfying, we believe it will be impossible to identify a general optimal reserve configuration due to current uncertainty in both biological parameters and selection of appropriate models. As an alternative perspective, we imagine a rather "hit and miss" approach, in which reserves of varying sizes will be examined for a wide variety of fish life histories and harvest regimes. Given this ad hoc approach, we ask, what patterns of reserve effectiveness are we likely to observe? We define "effectiveness" in ways that relate to what might be monitored following reserve establishment, with the idea that public support for marine reserves will be strengthened if one can measure increases in density and yield.

\section{Methods}

\subsection{Model description and parameter selection}

We used a simple model with adult density-dependence, fishing, larval dispersal into a common pool, larval loss from this common planktonic pool, and spatial structure. By spatial structure we mean that the total area is 
divided into two regions, a reserve acting as a "no take zone" and an unprotected region where the population is fished. Using this model, the chronology of events in the model is as follows. A fraction $\mathrm{f}$ of adults is fished in the unprotected area. The fraction surviving competes and reproduces. The resulting number of larvae produced at year $t$ at both the reserve $L_{t}(R)$ and unprotected area $L_{t}(U)$ is given by the Ricker dynamics,

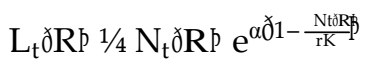

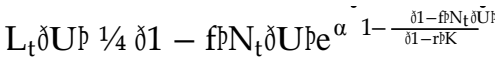

Here, $\alpha$ is the intrinsic growth rate for the population (such that $\exp ^{\alpha}$ gives the annual rate of increase when there is no density effect), $r$ is the fraction of the area set up as the reserve, and $\mathrm{K}$ is the carrying capacity of the total area (reserve plus unprotected). Next, a fraction $\mathrm{m}$ of the larvae produced in both reserve and unprotected areas migrates into a common pool, while the remaining fraction remain in their birthplace. Larvae in the dispersing pool are mixed in the plankton with a fraction $S$ of this planktonic pool being lost, while the remainder (1-S) recruit back to both the protected and unprotected areas at the beginning of next generation. The fraction of the common pool recruiting back to each zone depends on the zone's relative area. The number of next-generation adults in the reserve, $\mathrm{N}_{\mathrm{t}+1}(\mathrm{R})$, and the unprotected area, $\mathrm{N}_{\mathrm{t}+\mathbf{1}}(\mathrm{U})$, can be written as:

$\mathrm{N}_{\mathrm{tp} 1} \mathrm{RP}^{1 / 4} \gamma_{1}-\mathrm{mPL}_{\mathrm{t}} \mathrm{R} \mathrm{p}$ p m r r $1-\mathrm{Sp}$

$$
-ð \mathrm{~L}_{\mathrm{t}}{ }^{\mathrm{R} P} \mathrm{p} \mathrm{L}_{\mathrm{t}} \mathrm{U}^{\mathrm{UP}}
$$

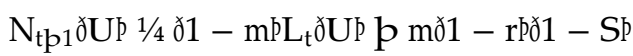

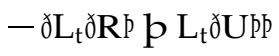

Our model is a discrete time model of a population with non-overlapping generations. This model applies explicitly to non-overlapping generations, or phenomenologically to any population whose dynamics can be approximated as a discrete recursion (Levin and Goodyear, 1980; Ludwig and Walters, 1985; Ludwig, 1998). The approximation for overlapping generations and age-structured populations is best if at a stable age distribution. For example, Levin and Goodyear (1980) show that a simple Ricker model approximates a simple age-structured model for striped bass. These examples are based on the premise that the intrinsic rate of increase is simply the maximum observed annual increment in populations. Simple Ricker-type models are also very common in the literature because they are simple to work with yet generally capture the most important features of fish population dynamics (Levin and Goodyear, 1980; Ludwig and Walters, 1985; Ludwig, 1998; Mangel, 1998). Second, such models can describe more complicated situations because they are quite general, and can easily be extended to a situation with overlapping generations. In particular, assuming stable agestructure, the net reproductive rate $R$, the basic reproductive rate $R_{0}$ (a parameter easily estimated from a cohort life table), and the generation time $\mathrm{T}$, are related by the relationship $\alpha=\ln R=\ln R_{0} / T$, where $\alpha$ is the intrinsic rate of annual increase (Begon et al. 1990, pp. 148-151). If generations are discrete, $T=\mathbf{1}$ and $R=R_{0}$. If $R_{0}$ ffi 1 , and/or there is little variation in generation time, a good approximation to $\alpha$ is $\ln R_{0} / T_{c}, T_{c}$ being the cohort generation time, which can again be obtained from a cohort life table (May, 1976). Thus, we believe that the model is general enough to approximate a large number of marine, commercially exploited species.

One of the most daunting challenges facing studies of marine reserves is understanding the influence of dispersal into and out of the reserve. The prominence of marine life histories with larvae that develop in the plankton means that dispersal of juveniles will be a key component of the connectivity between marine populations. Based on the life history for marine species considered, our model assumes that the primary mechanism of movement is larval dispersal. This is a common assumption in marine models (e.g. Mann et al., 1995; Holland and Brazee, 1996; Sladek-Nowlis and Roberts, 1999; Lindholm et al., 1998).

In this paper we explore the models described by Eqs. $(1 \mathrm{a}, \mathrm{b})$ and $(2 \mathrm{a}, \mathrm{b})$ for 5120 parameter combinations (Table 1). Our analyses were restricted to parameter values within the stable domain, so that results would be independent of initial conditions. We simulated the dynamics of our model without any reserve until densities converged to their steady state. Next we simulated the establishment of the reserve and allow for convergence again. We focused on the change in equilibrium densities and harvest following the establishment of a reserve, as well as the number of generations required to attain an equilibrium. In this paper we consider a population extinct when its density is less than one individual.

In our model, conservation effectiveness (CE) is defined as the ratio of equilibrium population density within the reserve relative to the density in the same area prior to establishment of the no-take zone. Yield effectiveness (YE) is the ratio of equilibrium annual number of fish caught outside of the reserve relative to the annual total catch over the entire space prior to any no-take restrictions. We recognize that multiple definitions of reserve effectiveness could be considered, but have chosen our two measures to capture the two most prominent goals in marine reserve theory: conservation or sustainable harvest (Gerber et al., 1999). 
Table 1

Parameter values and combinations used in simulation model [Eqs. (1a, b) and (2a, b)]

\begin{tabular}{llr}
\hline Parameter & Values considered & $\begin{array}{r}\text { Cumulative number of } \\
\text { parameter combinations }\end{array}$ \\
\hline Population Growth Rate $(\alpha)$ & $0.5,1.0,1.5,2.0$ & 4 \\
Fishing pressure (f) & $0.1,0.35,0.6,0.85$ & 16 \\
Migration Rate (m) & $0.1,0.35,0.6,0.85$ & 64 \\
Carrying Capacity (K) & $250,500,750,1000$ & 256 \\
Larval Loss (S) & $0.2,0.9$ & 512 \\
Reserve Area (r) & $0.05,0.15,0.25,0.35,0.45,0.55,0.65,0.75,0.85,0.95$ & 5120
\end{tabular}

\subsection{Analysis of model output}

We measured efficacy in both reserved and unprotected areas for CE, and found a strong correlation between these two metrics $\left(r^{2}=0.98\right)$. Thus, with the goal of parsimony, we focus on efficacy in the protected area. In addition, YE includes fish caught over the entire area, and thus describes effects on populations outside of the protected area. Because the equilibrium values for $\mathrm{CE}$ and YE are not attainable analytically (the equations involve transcendental functions of the variables in an essentially non-algebraic way), we could not analyze the model directly. Thus we adopt a simulation approach for our analysis, which comprises four ingredients. First, we ask what suites of model parameter combinations lead to the populations going extinct, and identify the one or two model parameters that govern whether or not a population goes extinct. Second, for some situations, it is possible for the creation of a reserve to have no positive effect on the target populations (either in terms of CE or YE). We use a multivariate analysis to understand when this is the case, and examine this result in light of the population dynamics implied by the model represented by Eqs. $(1 \mathrm{a}, \mathrm{b})$ and $(2 a, b)$. Finally, we use multiple linear and polynomial regressions to examine how well model parameters can explain variation in conservation and yield effectiveness.

\section{Results}

Before focusing on our analyses, let us first consider how fast the populations converge to a new steady state after reserve establishment. This is a non-trivial question since managers might be concerned not only with the long-term effectiveness of the reserve, but also with the time horizon necessary to achieve such benefits. We calculated convergence times for each parameter combination that did not lead to extinction. Convergence time was defined as the number of time-steps elapsed before the difference between the density at that particular time-step and the long-term density was less than one individual. Fig. 1, which displays the

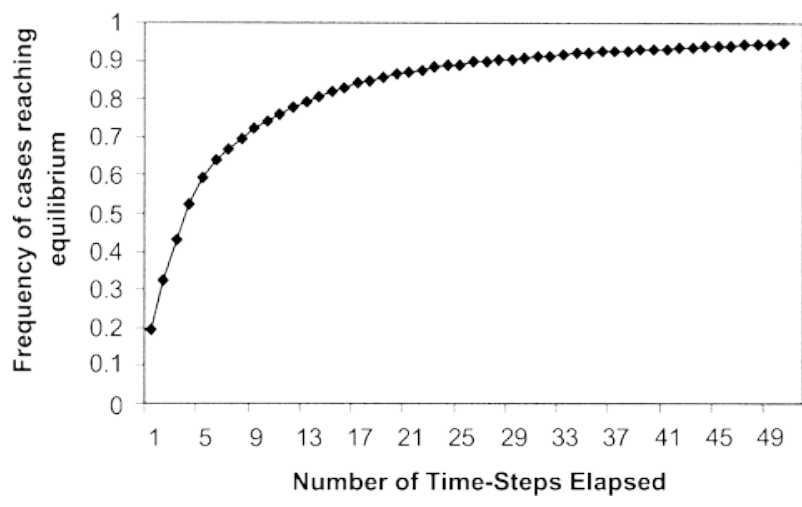

Fig. 1. Cumulative frequency of times to reach steady state after reserve establishment for 3156 parameter combinations. Convergence is defined as less than a one integer difference between density in a particular year and the long-term density.

cumulative frequency of time to convergence after reserve establishment, shows that in $19 \%$ of the cases, convergence occurred in a single generation. Furthermore, $74 \%$ of the simulations converge within a time horizon of less or equal to 10 time-steps. The few cases that took longer to converge involved parameters that tended to show dampening population cycles before settling into an equilibrium (i.e. high $\alpha$ ). This rapid convergence is pertinent to interpreting the results described below.

\subsection{What factors best predict the extinction of target populations?}

We used discriminant function analysis to identify multivariate parameter combinations that best predicted cases leading to extinction. Recall that we simulated the dynamics of our model without any reserve (fishing takes place in both $\mathrm{R}$ and $\mathrm{U}$ ) until densities converge to their steady state and then simulate the establishment of the reserve and allow for convergence again. The parameter combinations displayed in Table 1 represent 5120 unique simulations, of which 2712 persisted and 2408 went extinct in the absence of a reserve. The groupings for our discriminant function analysis were "extinct" versus "not extinct", with the model parameters $(\alpha, f, m, K, S)$ as potential classifying variables. 
We performed a stepwise discriminant function analysis in which we examined the ability of any single parameter, or combination of two parameters to classify cases as "extinct" or "not extinct". With harvest alone, we could classify $75 \%$ of the cases correctly, and with harvest and population growth as a pair we could classify $84 \%$ correctly (Tables 2 and 3, Fig. 2). Thus, harvest and population growth had the strongest influence on whether or not a harvested population went extinct.

\subsection{What factors best predict whether establishing a} reserve will have any positive effect?

In several simulations the establishment of a reserve did not enhance the target population. We used stepwise discriminant function analysis to identify cases for which reserve effectiveness indices were less than one. Using only harvest rate and population growth, $85 \%$ of cases considered were correctly classified with regard to conservation effectiveness being positive $(C E>1)$ versus ineffective ( $\mathrm{CE}<1$, Tables 4 and 5). For $\mathrm{YE}$, by using harvest rate alone it is possible to predict with $76 \%$ accuracy which simulations yield no measurable harvest benefit (Tables 4 and 5, Fig. 3). None of the other pairwise parameter combinations improve the classification beyond the classification achieved by harvest alone.

Table 2

Group means of discriminant function analysis for multivariate parameter combinations leading to extinction ${ }^{\mathrm{a}}$

\begin{tabular}{lcc}
\hline & Not Extinct & Extinct \\
\hline Population growth rate $(\alpha)$ & 1.48 & 0.99 \\
Migration $(\mathrm{m})$ & 0.41 & 0.55 \\
Carrying Capacity $(\mathrm{K})$ & 625.7 & 624.2 \\
Fishing pressure (f) & 0.32 & 0.65 \\
Larval Loss (S) & 0.48 & 0.63 \\
Number of cases & 2712 & 2408 \\
\hline
\end{tabular}

a Each value represents the average of all parameter values for categories "extinct" and "not extinct". Ninety-three percent of cases were correctly classified using five model parameters.

Table 3

Outcome of classification using discriminant function analysis for parameter combinations leading to extinction using all possible singlefactor discriminators, and the top four (in terms of percent correctly classified) pairwise combinations of parameters

\begin{tabular}{ll}
\hline Parameters used to discriminate & Percent correctly classified \\
\hline Harvest $(\mathrm{f})$ & 75 \\
Growth Rate $(\alpha)$ & 69 \\
Carrying Capacity (K) & 50 \\
Larval Mortality (S) & 61 \\
Migration Rate (m) & 62 \\
Harvest and Growth Rate & 84 \\
Harvest and Migration Rate & 77 \\
Harvest and Carrying Capacity & 75 \\
Harvest and Larval Mortality & 75 \\
\hline
\end{tabular}

Thus, harvest rate is the most important parameter for predicting effectiveness in terms of YE and CE, with higher harvest rates implying a great chance than reserves will be effective by in terms of either conservation or yield metrics. As expected, the second most important discriminating variable differs between CE and YE. For CE the key to conservation effectiveness is population growth rate; for $\mathrm{YE}$, life history parameters have a negligible influence on reserve efficacy as compared to harvest.

\subsection{How correlated are yield effectiveness and conservation effectiveness?}

In few cases did reserves result in an increased yield (7.5\% of cases) compared to increased density for $56 \%$ of cases. Although there was a positive correlation between $\mathrm{YE}$ and CE $\left(\mathrm{r}^{2}=0.463, \mathrm{P}<0.0001\right.$, Fig. 4a), that correlation was driven almost entirely by the few cases (204) where both CE and YE were greater than one. If one looks only at the vast majority of parameter combinations $(92.5 \%)$ that did not lead to extinction and did not increase yield, this correlation declined substantially $\left(\mathrm{r}^{2}=0.0001, \mathrm{P}=0.4\right.$, Fig. $\left.4 \mathrm{~b}\right)$. In other words, although it is impossible to achieve an increased level of harvest without also increasing density inside the reserve, it is possible to find many scenarios with elevated density inside the reserve areas (CE), and no concomitant or proportional increase in harvest (YE).

\subsection{When reserves are established, what system attributes correlate best with their effectiveness?}

Reserve effectiveness is likely to vary with exploitation rate, life history (reproductive rate, migration rate, larval mortality, carrying capacity), and reserve area. Here our question is, "amidst the total mix of model parameters, how does reserve area impact CE and YE?". In our previous discriminant function analysis, our response variable was binary (effective or not effective); here our response variable is continuous. In particular, we used multiple linear and polynomial regressions to examine the relationship

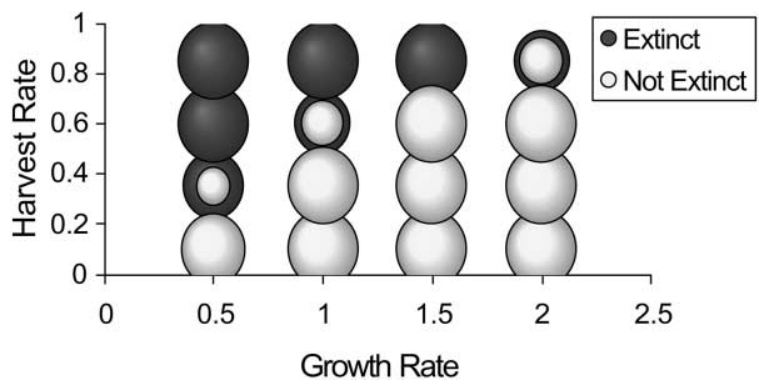

Fig. 2. Distribution of extinctions for combinations of growth rate and harvest rate, where categories represent the two outcomes: extinct versus not extinct. 
Table 4

Group means of discriminant function analyses for classifying simulation results into one of two groups: no benefit to reserve (reserve effectiveness $<1$ ) or a measurable benefit (reserve effectiveness $>1)^{a}$

\begin{tabular}{|c|c|c|c|c|}
\hline & Not Effective (CE) & Effective (CE) & Not Effective (YE) & Effective (YE) \\
\hline Population growth rate $(\alpha)$ & 1.817 & 1.211 & 1.49 & 1.338 \\
\hline Migration $(\mathrm{m})$ & 0.292 & 0.349 & 0.415 & 0.289 \\
\hline Carrying Capacity (K) & 625.00 & 625.00 & 624.6 & 634.8 \\
\hline Fishing pressure (f) & 0.400 & 0.409 & 0.304 & 0.573 \\
\hline Larval Loss (S) & 0.433 & 0.514 & 0.480 & 0.461 \\
\hline Reserve Area (r) & 0.5 & 0.5 & 0.511 & 0.365 \\
\hline Number of cases & 1200 & 1516 & 2512 & 204 \\
\hline
\end{tabular}

a 87 and $77 \%$ of cases were correctly classified using CE and YE, respectively, for five model parameters.

Table 5

Outcome of classification using discriminant function analysis for parameter combinations resulting in effective vs. not effective reserves using all possible single-factor discriminators, and the top four (in terms of percent correctly classified) pairwise combinations of parameters

\begin{tabular}{ll}
\hline Parameters used to discriminate & Percent correctly classified \\
\hline CE & 73 \\
Growth Rate $(\alpha)$ & 54 \\
Larval Loss (S) & 53 \\
Harvest (f) & 50 \\
Carrying Capacity (K) & 50 \\
Migration Rate (m) & 49 \\
Reserve Area (r) & 85 \\
Growth Rate and Harvest & 79 \\
Growth Rate and Larval Mortality & 76 \\
Growth Rate and Migration Rate & 73 \\
Growth Rate and Reserve Area & \\
& \\
YE & 76 \\
Harvest (f) & 68 \\
Growth Rate ( $\alpha$ ) & 50 \\
Carrying Capacity (K) & 42 \\
Larval Loss (S) & 44 \\
Migration Rate (m) & 61 \\
Reserve Area (r) & 76 \\
Harvest and Migration Rate & 76 \\
Harvest and Carrying Capacity & 76 \\
Harvest and Larval Mortality & 73 \\
Harvest and Reserve Area & \\
\hline
\end{tabular}

between life history attributes and harvest regimes on $\mathrm{CE}$ and YE. For the polynomial regression we included quadratic terms for migration rate and reserve area because previous models have suggested a nonlinear impact of these variables on reserve effectiveness (e.g. Polachek, 1990; DeMartini, 1993). We did not include quadratic terms for all of the other variables, because our question was not whether one could write a statistical model to describe variation in $\mathrm{CE}$ and $\mathrm{YE}$, but whether relatively a simple model would describe the observed variation. Our rule of thumb for adding new terms to the model was $>1 \%$ increase in variation explained as a result of that term. The results, summarized in Table 6, indicate that simple relationships
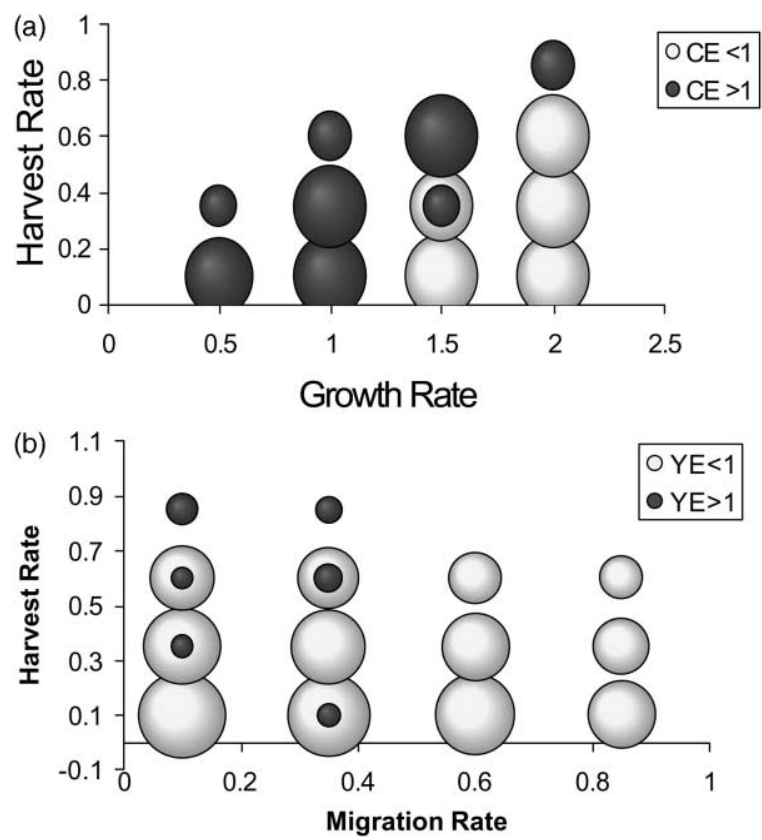

Fig. 3. Distribution of indices for reserve effectiveness (RE) for cases that did not go extinct for (a) conservation efficacy, and (b) yield efficacy. The two categories represent $\mathrm{RE}>1$ and $\mathrm{RE}<1$.

between either YE or CE and model parameters do not exist. No model with less than five variables account for even one quarter of the variation in $\mathrm{CE}$ or $\mathrm{YE}$, even when nonlinear functions involving the size of the protected area or migration rate were incorporated. Nonetheless, the regression analysis does provide a few clear messages. First, harvest intensity was positively related to $\mathrm{CE}$, so harvest reduction will elevate population density in the protected area. Second, reserve size was negatively related to $\mathrm{YE}$ and the effect of reserve area was nonlinear (there was a tendency for total yield to decline as more area is protected; Table 4).

\section{Discussion}

Using a simple simulation model, we examined reserve effectiveness as a function of life history variation, fishing pressure, and reserve area for two different 

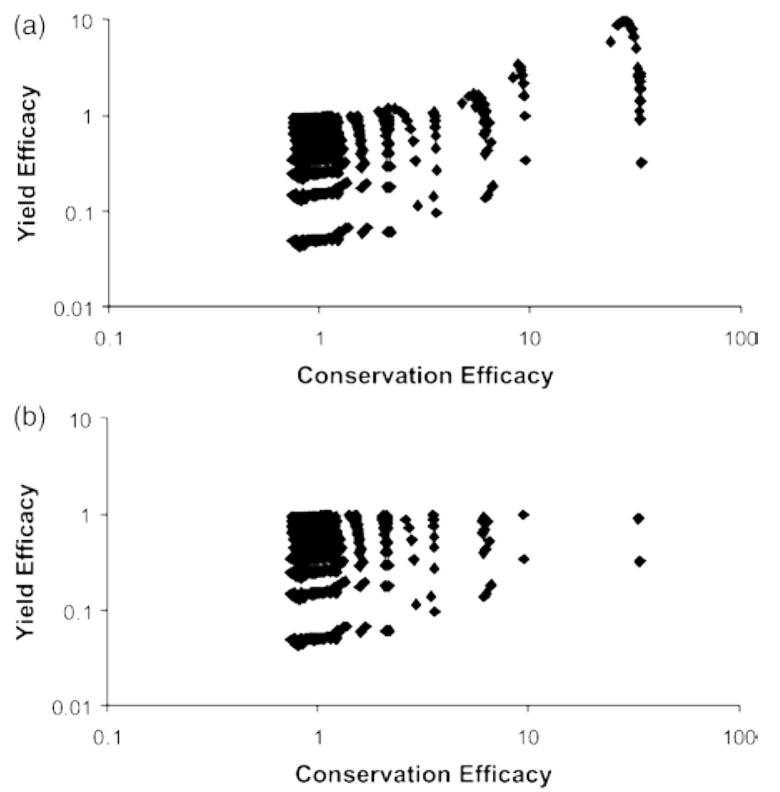

Fig. 4. Relationship between conservation and yield efficacy, (a) for all cases that did not go extinct, and (b) for all cases thad did not go extinct and where yeild efficacy is less than 1 .

measures of reserve efficacy. Our results demonstrate that marine reserves (1) can be effective at both preventing extinction and increasing density, and (2) rarely result in an increased level of total fishery yield. It is important to appreciate that for the vast majority (over $90 \%$ ) of life history and harvest regime contributions, the addition of a no-take zone did not enhance total number of fish caught. Importantly, like most other models of marine protected areas to date, our model was deterministic (Gerber et al., 2001). We anticipate that the greatest "harvest benefits" for no-take zones will emerge only after including a highly variable environment. Risk of over-exploitation may be misrepresented with deterministic models because of not adequately factoring in that variability, as well as poor estimates of fish abundances and demographic parameters. However, even though our model is unrealistic because it is not stochastic, it provides a cautionary tale. Our work departs from many previous studies using marine reserve models in that it seeks to describe how reserve effectiveness varies across a wide range of parameter values and reserve areas, while most other theory seeks to identify some "optimum solution" to the question of reserve design. In practice rarely do we have the luxury of information required for the establishment of an optimal reserve. Thus while optimal reserves are possible in theory, a more practical question might be, given a particular reserve area, how likely are we to see a noticeable improvement in the system following the establishment of a marine reserve? The answer is that reserve effectiveness measured by an enhanced fish density inside the reserve will be most apparent when
Table 6

Stepwise multiple polynomial regression for CE and YE for 2712 output data points ${ }^{\mathrm{a}}$

\begin{tabular}{lccc}
\hline & Coefficient & $\mathrm{r}^{2}$ & P value \\
\hline Conservation effectiveness & & & \\
Fishing Pressure (f) & 10.56 & 0.15 & $<0.0001$ \\
Population Growth Rate $(\alpha)$ & -2.07 & 0.18 & $<0.0001$ \\
Larval loss (S) & 1.44 & 0.19 & $<0.0001$ \\
& & & \\
Yield Effectiveness & & & \\
Reserve Area (r) & -2.4 & 0.13 & $<0.0001$ \\
Fishing Pressure (f) & 1.29 & 0.19 & $<0.0001$ \\
Population Growth Rate $(\alpha)$ & -0.35 & 0.22 & $<0.0001$ \\
(Reserve Area) $^{2}$ & 1.60 & 0.23 & $<0.0001$ \\
\hline
\end{tabular}

a Quadratic terms were only included for two predictor variables: fraction of area in no-take protected area, and migration rate. The remaining variables were assumed to be linear. The predictor variables were listed in the order in which they were entered, with their coefficients indicating the direction of their influence for the final model (all terms), and the cumulative $r^{2}$ values summarizing the percent of variation explained with the corresponding variable and all variables listed above it entered into the predictive model. The r-squared values indicate the proportion of the variation in the effectiveness metrics that can be explained by a simple statistical model. The addition of each term increased the $\mathrm{r}^{2}$ value by at least 0.01 (i.e. at least an additional $1 \%$ of the variation explained).

population growth rate is low and fishing pressure is high (Tables 4 and 6), or when over-exploitation is greatest. This is an expected result and agrees with results from prevous marine reserve models (e.g. Polachek, 1990; DeMartini, 1993; Quinn et al., 1993; Sladek Nowlis and Roberts, 1999). Less obvious is the few parameter combinations that produce enhanced total annual catches of fish (Table 5). Marine protected areas are most likely to increase total catch when harvest is very high (thus over-fishing is likely), and when the reserve area is not too large. We predict that as environmental variability is incorporated into the analysis, along with uncertainty in parameter estimation, the region in parameter space where increased yields are observed will expand, and will embrace larger reserve sizes.

Our results reinforce the idea that fishing pressure plays a dominant role in dictating the effectiveness of marine reserves-both as measured by the conservation of populations inside the reserve, and the possible increase in total yield. Clearly aspects of life history can modify this effect. As a practical message, we suggest that fishery management would be best served by obtaining improved estimates of total populations size and fraction of population harvested, as opposed to building more elaborate models that embrace a great diversity of life history types. The one exception to this is migration rate, which does help discriminate among cases in which a reserve enhances total yield (YE $>1)$ versus does not improve YE (Table 5). 
Simple models rarely represent reality faithfully enough to become management tools. But they can serve as steps along the way to building more realistic models that are more directly applicable-especially when they give guidance on what are the most important parameters or patterns on which to focus. Population growth rate and fishing pressure dominate as explanatory factors underlying the effectiveness of reserves following establishment. This suggests that as we move to multispecies models in more complicated spatial networks, we may be able to keep our representation of life history variation simple and focus on fishing pressure and population growth rate as the critical factors to be represented in these models.

\section{Acknowledgements}

This work was conducted as part of the "Developing the Theory of Marine Reserves" Working Group supported by the National Center for Ecological Analysis and Synthesis, a Center funded by NSF (Grant \#DEB94-21535), the University of California-Santa Barbara, the California Resources Agency, and the California Environmental Protection Agency. Support was provided to JB by a grant from the Spanish Ministry of Science and Technology (B05200-1366-C02-02). We thank G.C. Attwood, L. Botsford, S. Palumbi and an anonymous reviewer for insightful comments on this manuscript.

\section{References}

Allison, G.W., Lubchenco, J., Carr, M.H., 1998. Marine reserves are necessary but not sufficient for marine conservation. Ecological Applications 8 (1), 79-92.

Begon, M., Harper, J.L., Townsend, C.R., 1990. Ecology, 2nd Edition. Blackwell Science, Boston.

Beverton, R.J.H., Holt, S.J., 1957. On the dynamics of exploited fish populations fishery investigations. Fisheries Investigations, London (series II) 19, 1-533.

DeMartini, E.E., 1993. Modeling the potential for fishery reserves for managing Pacific coral reef fishes. Fishery Bulletin 91 (3), 414-427.

Gerber, L.R., Botsford, L., Hastings, A., Possingham, H., Gaines, S., Palumbi, S., Andelman, S., 2001. Population models for reserve design: a retrospective and prospective synthesis. Ecological Applications (Special Issue on Marine Reserves), in press.

Guenette, S., Lauck, T., Clark, C., 1998. Marine reserves: from Beverton and Holt to the present. Reviews in Fish Biology and Fisheries 8, 251-272.

Holland, D.S., Brazee, R.J., 1996. Marine reserves for fishery management. Marine Resource Economics 11, 157-171.

Lauck, T.C., Clarke, W., Mangel, M., Munro, G.R., 1998. Implementing the precautionary principles in fisheries management through marine reserves. Ecological Applications 81 (1), S72-S78.

Levin, S.A., Goodyear, C.P., 1980. Analysis of an age-structured fishery model. Journal of Mathematical Biology 9 (3), 245-274.

Lindholm, J., Ruth, M., Kaufman, L., Auster, P., 1998. A modeling approach to the design of marine refugia for fishery management. In: Linking Protected Areas with Working Landscapes. Science and Management of Protected Areas Association, Wolfville, Nova Scotia, pp. 138-150.

Ludwig, D., 1998. Management of stocks that may collapse. Oikos 83, 397-402.

Ludwig, D., Walters, C.J., 1981. Measurement errors and uncertainty in parameter estimates for stock and recruitment. Canadian Journal of Fisheries and Aquatic Science 38, 711-720.

Mangel, M., 1998. No-take areas for sustainability of harvested species and a conservation invariant for marine reserves. Ecology Letters 1, 87-90.

Mann, A., Law, R., Polunin, N.V.C., 1995. Role of marine reserves in recruitment to reef fisheries: a metapopulation model. Biological Conservation 71, 197-204.

May, R.M., 1976. Estimating r: a pedagogical note. The American Naturalist 110, 496-499.

Myers, R.A., Mertz, G., 1997. The limits of exploitation: a precautionary approach. Ecological Applications 8 (Suppl. 1), S165S169.

Polacheck, T., 1990. Year round closed areas as a management tool. Natural Resource Modeling 4, 327-354.

Quinn, J.F., Wing, S.R., Botsford, L.W., 1993. Harvest refugia in marine invertebrate fisheries: models and applications to the red sea urchin, Strongylocentrotus franciscanus. American Zoologist 33, 537-550.

Sladek Nowlis, J.S., Roberts, C.M., 1999. Fisheries benefits and optimal design of marine reserves. Fishery Bulletin 97 (3), 604-616. 